\title{
Enterprise integration - engineering tools for designing enterprises
}

\author{
Peter Bernus ${ }^{1}$ and Laszlo Nemes ${ }^{2}$
}

\section{$1 \quad$ INTRODUCTION $^{3}$}

With the globalisation of economies, enterprises are more viewed as 'products' themselves. This is quite obvious for green field companies as they are designed, built and put into operation as the customer requires. The product view, however, is also true for already existing enterprises. New business priorities have to be analysed, changes have to be specified, designed and carried out, and the design of an enterprise may take a considerable effort.

Business, manufacturing practice, organisational structure, information infrastructure keep changing to provide the most effective framework for the core business of an agile enterprise. Therefore enterprises should often be re-designed, and these modifications could be significant.

To design enterprises and manage them through their entire life-cycle we need fundamental principles of design, methodologies based on these principles, as well as supporting tools. Those who build machines, electrical devices or business software products have been using design and analysis tools for a long time. These tools are product oriented, being developed with a certain class of product and a certain type of designer-user in mind. An enterprise as a design object includes all equipment and organisation needed to implement the complete lifecycle of its products, from product innovation, through design and production to distribution and after sales service, and recycling.

Tools needed for enterprise integration are not just more complex but substantially different from design tools intended for other products. This difference appears on two accounts:

- The nature of the 'product' (i.e. the enterprise) is more heterogeneous then any of the product classes mentioned above

- The disciplinary mix of the 'designers' involved has an additional dimension. In concurrent engineering situations it is engineers of various disciplines who are involved in the design process, while in enterprise engineering the 'designers' also belong to various levels of the

1.Griffith University, School of Computing and Information Technology

2.CSIRO Division of Manufacturing Technology

3.This research was supported by CSIRO priority funding. 
hierarchy and have a wide range of power status, therefore the support for mutual understanding and group dynamics in the process of enterprise design becomes an even more serious requirement than in usual collaborative design.

\section{THE RELATIONSHIP OF ENTERPRISE INTEGRATION TO OTHER APPROACHES}

Global business entities or enterprises need to constantly renew themselves. This process of renewal needs continuous and efficient design and redesign of the enterprise so that it keeps achieving its business goals successfully and in a sustainable manner.

A global enterprise may or may not be incorporated, but in either case it needs to be designed to fulfill its business aims. As business aims change so should the implementing enterprise and the change should be dynamic and quick. The change process involves change in several aspects of an enterprise, such as its management, control and information flow, organisation and production technology. This process of change itself needs to be properly initiated, managed and executed.

It is necessary to develop a system of methods which can be utilized to manage the total life-cycle of a global enterprise from its conception through its implementation and constant renewal together with these methods being supported by a system of tools as needed.

Enterprises considered may be of any kind: continuous process plant installations (e.g. chemical), discrete part manufacturing enterprises (producing components, sub-assemblies, complex machines or one-of-a-kind objects) or those in service (e.g. hospitals, hotels, government organisations).

The challenge is to provide management, and all involved parties, with methods and suitable accompanying tools that allow the business design or redesign tasks to be carried out with great expediency.

The product of the enterprise design task is a renewed enterprise, consequently enterprises in this context are considered to be products themselves as has been explained in the introduction. Similar to families of manufactured products enterprises also share common design elements so that the methods and tools above will be reusing tested component designs. This ensures the speed and quality of enterprise design needed by business. Even if an enterprise is designed from scratch (green field installation) the components that will be used must be previosly tested ones.

The methods for enterprise design are intimately linked with product life-cycle management methods, because the aim of the designed enterprise is to implement the life-cycle, or value chain, of its products.

Enterprise integration methodologies and tools presuppose that there are significant opportunities to be tapped by the enterprises using these methodologies. Such opportunities may be technological (in the production technology or in the control- and information technology), or in the management or organisational approach used. Enterprise integration methodologies will then ensure that opportunities offered by new techniques are turned into working systems, blended ('integrated') into the entire enterprise's business process. 
Some examples of opportunities that have been recently proposed (and by the lack of a comprehensive methodology have delivered mixed results):

- Business process reengineering: here the significant opportunity identified is that customer centered business processes can deliver more efficient operations ( $\mathbf{M}$ Hammer and $\mathbf{J}$ Champy, 1993). Enterprise integration methodologies and accompanying tools are needed to ensure consistent results when trying to exploit a reengineering opportunity.

- Virtual enterprise: the concept is based on the realisation of a significant opportunity that it is possible to design and operate a not-incorporated enterprise around the value chain of a product, i.e. to design the product's value chain across organisational boundaries (Goldman et al, 1995). This allows dynamic and quick establishment of 'virtual' enterprises in which, however, there exists a global and well controlled product life-cycle. The technique presupposes the mastery of the methodology and the availability of those modeling techniques which this task group investigates.

- Concurrent engineering, simultaneous engineering, around the clock engineering: the significant opportunity to be capitalised on is the availability of technologies and distributed teamwork techniques that enable a highly parallelised design and implementation process with drastic cuts in the time-to-market of products. It is then the enterprise design methodologies and accompanying tools that enable an enterprise (or virtual enterprise) to capitalise on the concurrent techniques in a systematic way.

- Downsizing: the significant opportunity recognised is that certain functions do not form the core business of an enterprise and therefore can be separated from it and operated as an independent business. This makes the use of resources more efficient, as long as core (strategic) tasks are retained.

- Information technology solutions for the support of enterprise integration (distributed, network-based data, process, communication etc. systems): these technologies are widely believed to be prerequisite to the integrated information flow that enterprise integration aims to achieve,because they create either new opportunities not available before or enable more efficient service delivery.

E.g. a significant opportunity due to IT solutions is the use of 'executable enterprise models', or 'model enactment' -- which ensure that business process models and business process execution are tightly connected, thereby eliminating the phenomenon of business process models becoming quickly obsolete. In this approach business process models are 'live' and modifications to them will affect the actual processes. Conversely all important current business processes are represented and available for management to investigate/ analyse.

\section{BUSINESS PROCESS PERSPECTIVE}

The traditional concept of computer integrated manufacturing has been aiming to provide computer assistance for various elements of product development, manufacturing processes and management activities. Integration in CIM in the eighties meant to 'link' one computer aided activity to another to provide smooth information and material exchange among them. 
Enterprise integration goes a big step beyond that: it asks questions about the core business processes in a organisation before attempting to design or redesign existing ones. Only after that does EI investigate how to perform processes better using a mix of technology and human resources.

In such a context Enterprise Integration captures and describes the enterprise's mission, objectives, strategies, goals and constraints, organisational structures, resources, and all management and business processes in the value chain of products. It specifies the business process requirements, identifies the solution options, presents alternative designs and provides implementation paths at strategic, tactical and operational levels.

The entire business process is studied and described in terms of two inter- linking networks.

- The network of business processes which form the product value chain

- The network of business processes which encompass the decisional- and management functions of the enterprise.

These two networks are then implemented in three subsystems: the control-information system, the productions system and the human organisation. Enterprise integration methodologies include the methods and provide the framework for the design of the processes within and among these three systems. The framework in which enterprise integration methodologies work is that of enterprise reference architectures. A substantial departure from the mechanistic information system design principles is the use of co-ordination theory (Kling, 1991) allowing a less mechanical design of human-executed business processes.

\section{4}

EXTENDED, VIRTUAL ENTERPRISES

The traditional view of an enterprise is that of a hierarchical organisation. In big companies with diversified business profiles an enterprise may mean a branch office or a plant that operates as an independent business unit.

Very few companies however design and manufacture all components in house. In fact most of the agile enterprises co-operate with a large number of suppliers and subcontractors. Components and subassemblies are therefore manufactured outside the premises of the product vendor. More recently products are being designed in close cooperation with partners in the supply chain; some suppliers are in strategic relations with a number of product vendors using concurrent engineering methods (Cutkosky et al., 1993).

When designing the business processes of an enterprise one needs to address all value adding activities through all supply chains irrespective of whether they cut across organisational boundaries or not. E.g. company ' $A$ ' could have business processes of which a part is being performed outside the physical boundaries of the company. The value chain is not necessarily hierarchical, but there is no a priori superior structure for cooperation among units of business. This aspect of the enterprise is also to be designed and not taken as given. Design tools therefore should be able to help construct distributed cooperative, extended enterprises (Goldman $e t$ al, 1995).

The above help may take the form of business decision support, like 'make or buy' or 'make or have it made' which are certainly key factors when defining the extended integrated 
enterprise. Business activities (focus) may change quickly and so should change the business process as well. The dynamism, the time dimension of the enterprise design and implementation through the whole life-cycle, is critical and will be even more critical in the future. Virtual or extended enterprises will be formed and dissolved as quickly as the underlying business processes are changed. It is therefore an industry need to get design support for the design of (and experimentation with) such virtual enterprises.

\section{ENTERPRISE INTEGRATION METHODS}

Integrated manufacturing systems in the eighties and nineties were individually specified and designed. It took 1-1.5 years to summarise the customer's requirements and to develop a comprehensive set of specifications. These specifications were never complete and contained undetected contradictions. Because of the lack of formal descriptions, of trained CIM engineers proficient in building feasible CIM models, and the lack of integrated, capable modelling tools, it was impossible to model the expected functions and it took a long time to test and modify these early CIM systems. Early CIM systems therefore were custom built, integrated - but monolithic; once they were installed it was extremely difficult to update and to modify them. As design methodologies became more sophisticated, the design phase became shorter, but still all systems were individually made and crafted. It became obvious that integrated enterprises should not be tailor made but should be manufactured like any other industrial product. Enterprises should be designed for their whole life-cycle.

There are many - independently developed - techniques which are ready to be used either in the design or in the implementation phases of enterprise integration.

ESPRIT projects have developed integration architectures, modelling tools, and their industrial applications. The most important results have been the Computer Integrated Manufacturing Open System Architecture (CIMOSA) project, its supporting modelling tools and its validation in industrial environments, (CIMOSA, 1994). These results are restricted to various industrial consortia in Europe, but some of these are accessible to researchers through the CIMOSA Association.

The GRAI/LAP laboratory of the university of Bordeaux has developed a framework and modelling tools to support enterprise integration (Doumeingts, 1992). They are keen to prove their results in practice and have many industrial references.

The Purdue Consortium has developed an engineering-oriented architecture and associated implementation methodology. The architecture was published as the Purdue Enterprise Reference Architecture (Williams, 1992) and its Implementation Procedures Manual (not yet fully published in the open literature).

Toronto University Enterprise Integration Laboratory is developing a complete set of enterprise modelling tools to support any such architectures.

It is probably less well known that the presently widely used term of Business Process ReEngineering (Hammer and Champy, 1993), preferred by consultants and understood by company executives is just one, albeit important aspect of Enterprise Integration (EI). BPR, as it is abbreviated, focuses on the review and redesign of the core business process of an enterprise 
and it uses either common sense practice or professional tools offered by the EI community. There is a close cooperation between researchers of BPR and EI. What BPR does not address at this stage is the need to continuously engineer the business process, instead of just re-engineering it once.

Concurrent Engineering traditionally has been viewed as a powerful method to reduce time to market. It incorporates both technological and human-organisational issues. When we consider that concurrent design activities will span the entire supply chain - in which case the product vendor and subassembly (component) suppliers will be simultaneously working on the design of the new product - then Concurrent Engineering is really just one facet of EI. It is a complex method to carry out cooperation between distributed engineering tasks.

\section{GENERIC ENTERPRISE REFERENCE ARCHITECTURE (GERAM)}

All these previously mentioned architectures and integration methods have produced many fine results, but they influenced each other very little. Consequently there was a need to compare and evaluate them and to combine the various methodologies and modelling techniques and to identify the missing tools. Based on the result of the investigations by an international (Task Force, 1995), the authors proposed a new Generic Enterprise Reference Architecture and Methodology (GERAM) (Bernus and Nemes, 1994) which defines a toolbox of concepts for designing and maintaining enterprises during their entire life-cycle. GERAM is therefore about those methods, models and tools which are needed to build integrated enterprises. The architecture is generic because it applies to most, potentially all types of enterprise and because it is a framework document allowing multiple individual reference architectures to coexist. This is significant because GERAM does not enter the architecture development area as a competitor, rather it is a mediator, or common framework.

\subsection{Requirements for a Generic Enterprise Reference Architecture and Methodology.}

The architecture should cover all activities necessary for designing, operating, maintaining and improving enterprises. Since architectures are the backbone of any methodology necessary to engineer the complex business processes, the architecture should cover the entire enterprise life-cycle. (Individual reference architectures in use in industry or in research may or may not satisfy this requirement).

There is a need for a consistent modelling environment leading to executable business processes, and some of them are manifest as business software. It is an industry need that requirements should directly lead to executable code ('business process enactment' (Bussler, 1995)). The ideal modelling environment should be modular so that alternative methodologies could be incorporated into it. There should not be any restrictions built into the modelling languages as to what methodologies they would be applied for. The modelling environment should be extensible (rather than be a closed set of models) and be permissive, leaving space for alternative modelling methods. (CIMOSA and TOVE are good examples) (Vernadat, 1995). 
A comprehensive and easy to follow methodology must be technically correct and easy to use by multi-disciplinary teams, in designing enterprises within budget, expected time frame and resource constraints. The enterprise integration methodology should also be open and expandable and it should be presented both in generic and special forms. The former ones contain the general rules and the latter ones contain more detailed rules and serve particular industry sectors. (E.g. the GIM methodology and PERA have many industrial applications).

The cost and the time frame of the enterprise engineering process should be kept low. Adoption of reusable, tested (and standard) models (building blocks) greatly help this process. Enterprise integration and engineering are complex processes and are carried out by groups of people. This should be supported by sound design theory and collaboration technology.

GERAM should have provision for unifying product- and process mangement, enterprise development, and strategic management. Any architecture that covers the scope of GERAM should tie and relate enterprise integration and enterprise engineering to the rest of the activities in the enterprise.

It is especially important to support an evolutionary path (migration) to integrated enterprises and the strategy for migration should be part of any methodology.

\subsection{The Structure of the Generic Enterprise Reference Architecture and Methodology}

To satisfy the requirements, the components of GERAM are the following:

\subsubsection{Generic Enterprise Reference Architecture (GERA).}

An architecture is a description (model, framework) that collects (systematises) all concepts necessary to describe the structure, the elements and their relationships and all relevant properties (part, functions, cost, time, resources, etc.) of a device, a system or an enterprise. GERA is the definition of the enterprise related concepts, with primary focus on the life cycle of the enterprise.

\subsubsection{Generic Enterprise Engineering Methodology (GEEM).}

This is a generic description of the process of enterprise integration. The methodology is a detailed process model with instruction on each step of the integration project. There may be several (competing or specialised) methodologies which cover the scope of GEEM.

\subsubsection{Generic Enterprise Modelling Tools and Languages (GEMT\&L).}

Engineering of the integrated enterprise is a highly sophisticated (multi-disciplinary management, design, and implementation) exercise, during which various forms of descriptions and models of the enterprise need to be created. More than one modelling language is needed to describe an enterprise.

\subsubsection{Tested Components To Build Enterprise Models}

(a) Generic Enterprise Models (GEMs) or Enterprise Models (EMs) 
Generic Enterprise Models capture the concepts which are common to all enterprises. The enterprise engineering process can therefore use them as tested components for building any new enterprise models.

Re-usable enterprise models capture some common parts of enterprises. They are components of a complete set of models. The GEMs (or EMs) can be used as tested building blocks in the EI process.

Typical systems of such models are also known as 'Type I Architectures' (Williams et al, 1994).

- Ontological theories (OTs) that describe (define) enterprise related modelling concepts. They can also be viewed as meta models because the facts and rules are describing the modelling languages themselves. Ontological theories capture the most basic properties of enterprise related concepts (function, structure, dynamics, costs) (Gruber 1993), much as naive physics theories do (Hayes, 1985).

(b) Generic Enterprise Modules (GMs).

These are products or standard implementations of components that are likely to be used in enterprise integration, either by an enterprise integration project or by the enterprise itself. Generic modules (software or hardware systems) can be configured to form more complex modules for the use of individual enterprises.

\section{ENTERPRISE INTEGRATION CLEARINGHOUSE}

This initiative intends to collect all information on the major projects on Enterprise Integration. It updates its databases on enterprise modelling languages, meta-models, and associated tools. It reports on developments and validations of the major enterprise reference architectures and other tools and infrastructures for enterprise integration. It contains information on events related to enterprise integration and mailing list relevant to the topic. The clearinghouse in Australia is maintained by Griffith University and it is accessible on the World Wide Web (http://www.cit.gu.edu.au/research/ei95/clearinghouse.html).

\section{CONCLUSION}

The GERAM architecture was presented to the IFIP/IFAC Task Force on Enterprise Integration Architectures. The international committee (including experts from the CIMOSA Association, the Purdue Consortium and GRAI/LAP Laboratory) discussed and evaluated the proposal. The committee endorsed GERAM as a baseline document which allows the mapping of existing architectures to the set of requirements that GERAM was based on.

GERAM in its matrix form (as it was developed by the authors) is a powerful research tool. Because of its complex graphical representation it is difficult to be used effectively by practitioners of enterprise (re) engineering. A more visual representation of the architecture is needed to fully exploit its practical value. Recently $\mathrm{T} J$ Williams proposed a set of three dimensional models to explain GERAM in more industrial terms. It is expected that the matrix form 
will be used by researchers for developments and the three dimensional model will be preferred by educators to introduce it into engineering practice.

\section{REFERENCES}

Bernus, P. and L. Nemes (1996) 'A framework to define a generic enterprise reference architecture', Int J CIM (to appear)

Bussler, Ch. (1995) 'Workflow management systems as enterprise engineering tools,' (this volume).

M.R. Cutkosky et al (1993) 'PACT an experiment in integrating concurrent engineering systems' IEEE Computer, 26, (1).

CIMOSA (1994) 'Open System Architecture for CIM' CIMOSA Association.

G Doumenigts, B Vallespir, M Zanettin and D Chen. (1992) 'GIM - a GRAI integrated methodology. A method for designing CIM systems' Report. GARI/LAP, University of Bordeaux, France..

M S Fox, (1992) 'The Tovie Project, towards a common sense model of an enterprise integration' in Enterprise Integration Modeling, Ch Petrie (ed) MIT Press. Cambridge, Mass, USA.

S L Goldman, R N Nagel and K Preiss (1995) 'Agile Competitors and Virtual Organisations' Van Nostrand Reinhold Publishing Inc.

M Hammer and J Champy (1993) The Re-engineering the corporation. Nicholas Braedly publishing, London.

Hayes,P. (1985) 'Second Naive Physics Manifesto' in Ronald J. Brachman and Hector J.Levesque (eds), Readings in knowledge representation, Los Altos, Calif., Morgan Kaufmann Publishers,. pp 467-485.

IFIP/IFAC Task Force (1995), Architectures for Enterprise Integration, P.Bernus, L. Nemes, T.J. Williams (eds), Chapman and Hall, London.

Kling, R. (1991). 'Cooperation, coordination and control in computer-supported work' Comm. ACM, 34(12), 83-88.

Vernadat F. (1995) 'Integrated Manufacturing Systems Engineering', Chapman and Hall, London.

TJ Williams. The Purdue Enterprise Reference Architecture. Instrument Society of America. Research Triangle Park, North Carolina, USA. 1992.

TJ Williams et al (1994) 'Architectures for Integrating Manufacturing Activities and Enterprises,' Computers in Industry, 24, (2-3) pp111-140.

T. R. Gruber (1993) 'A Translation Approach to Portable Ontology Specifications' Knowledge Acquisition, 2, pp 199-220. 